\title{
Liderança transformacional e a eficácia grupal: o papel mediador dos comportamentos de suporte
}

\author{
Transformational leadership and team effectiveness: The \\ mediator role of supportive behaviors
}

\author{
Carina Isabel Pereira PESSOA ${ }^{1}$ \\ ORCID iD 0000-0002-6789-733X \\ Isabel Dórdio DIMAS 2 \\ ORCID iD 0000-0003-4481-2644 \\ Paulo Renato LOURENÇO ${ }^{1}$ \\ ORCID iD 0000-0003-1405-3835 \\ Teresa REBELO ${ }^{1}$ \\ ORCID iD 0000-0003-3380-0840
}

\section{Resumo}

O objetivo deste estudo foi analisar o papel mediador dos comportamentos de suporte na relação entre a liderança transformacional e quatro critérios da eficácia da equipe (designadamente, o desempenho, a viabilidade, a qualidade da experiência grupal e os processos de melhoria). Conduziu-se um estudo empírico com uma amostra composta por 653 participantes de 117 equipes de diferentes setores de atividade (e.g., indústria, proteção civil, consultoria), onde foram testadas as relações previstas. Os resultados mostraram um efeito direto positivo da liderança transformacional nos comportamentos de suporte, assim como dos comportamentos de suporte na eficácia da equipe (nomeadamente, no desempenho, na viabilidade, na qualidade da experiência grupal e nos processos de melhoria). Além disso, foi igualmente identificado um efeito mediador dos comportamentos de suporte na relação entre a liderança transformacional e os quatro critérios da eficácia da equipe. Implicações para a investigação, para a prática profissional e pistas para futuras pesquisas foram apresentadas.

Palavras-chave: Eficácia; Equipes de assistência ao paciente; Liderança.

\begin{abstract}
The aim of the present study was to analyse the mediator role of supportive behaviours in the relationship between transformational leadership and four team effectiveness criteria: performance, viability, quality of group experience, and improvement processes. An empirical study was conducted with a sample of 653 participants from 117 teams from different professional practice sectors (e.g., industry, civil protection, and consultancy), in which these relationships were

1 Universidade de Coimbra, Faculdade de Psicologia e de Ciências da Educação. R. do Colégio Novo, 3001-802, Coimbra, Portugal. Correspondência para/Correspondence to: C.I.P. PESSOA. E-mail: <carina.p.pessoa@gmail.com>.

2 Universidade de Aveiro, Escola Superior de Tecnologia e Gestão de Águeda, Unidade de Investigação em Governança, Competitividade e Políticas Públicas. Aveiro, Portugal.

Artigo elaborado a partir da tese de C.I.P. PESSOA, intitulada "O papel mediador da resiliência e dos comportamentos de suporte". Universidade de Coimbra, Portugal.

$\boldsymbol{\nabla} \mathbf{v} \boldsymbol{\nabla}$

Como citar este artigo/How to cite this article

Pessoa, C. I. P., Dimas, I. D., Lourenço, P. R., \& Rebelo, T. (2018). Liderança transformacional e a eficácia grupal: o papel mediador dos comportamentos de suporte. Estudos de Psicologia (Campinas), 35(1), 15-28. https://doi.org/10.1590/1982-02752018000100003
\end{abstract}


tested. The results showed a positive direct effect of transformational leadership on supportive behaviours and on team effectiveness (including performance, viability, quality of the group experience, and improvement process). Furthermore, the present study also identified the mediator effect of supportive behaviours on the relationship between transformational leadership and the four team effectiveness criteria considered. Implications for research and professional practice were discussed, and suggestions for further studies were offered.

Keywords: Efficacy; Patient care teams; Leadership.

Com mais de cem anos de evidência teórica e empírica, a liderança é conceitualizada como um elemento essencial para um funcionamento organizacional e social eficaz, tendo o seu estudo suscitado o interesse de uma multiplicidade de investigadores (Day \& Antonakis, 2012). A liderança transformacional, enquanto estilo de liderança centrado na pessoa, surgiu na década de 80 do século XX como uma forma eficaz de dar resposta a um contexto cada vez mais global e competitivo (Bass, 1999). Bass (1985) definiu-a como um processo de influência, na medida em que os líderes e os seguidores experimentam uma troca significativa e construtiva, a fim de proporcionarem mudanças nos sistemas sociais e no contexto.

O estudo dos comportamentos de liderança grupal e do seu impacto ao nível dos resultados alcançados pelas equipes tem recebido, nos últimos anos, uma atenção significativa (Burke et al., 2006). Apenas recentemente, contudo, os investigadores dessa área começaram a estudar os mecanismos mediacionais que estão na base dessas relações (Zhu, Newman, Miao, \& Hooke, 2013). O presente estudo insere-se nessa linha e pretende contribuir para clarificar os efeitos da liderança transformacional na eficácia dos grupos de trabalho, tendo por base o modelo de eficácia Input-Mediator-OutcomeInput (IMOI) de llgen, Hollenbeck, Johnson e Jundt (2005), no qual a liderança pode ser vista como um antecedente da eficácia (input). O impacto da liderança transformacional nos resultados da equipe concretiza-se, quer de forma direta quer por via da relação que estabelece com variáveis intervenientes ou mediadoras, as quais poderão constituir processos grupais ou estados emergentes (Kozlowski \& Ilgen, 2006). No presente estudo, analisa-se o papel mediador dos comportamentos membros se apoiam voluntariamente (Aubé \& Rousseau, 2005) na relação entre a liderança transformacional e a eficácia grupal.

A eficácia da equipe, enquanto conceito multidimensional, pode ser compreendida e avaliada por diferentes critérios (Hackman, 2012). Na presente investigação, de modo a obter um diagnóstico o mais completo possível da eficácia grupal, foram considerados quatro critérios: o desempenho grupal, que se refere à quantidade e qualidade dos outputs produzidos pela equipe (Hackman, 1987); os processos de melhoria da equipe, que podem ser definidos como o grau em que os membros do grupo implementam soluções e eliminam deficiências com vista a melhorar os resultados de tarefa (Rousseau \& Aubé, 2010); a viabilidade da equipe, que pode ser entendida como a capacidade da mesma para se adaptar a mudanças internas e externas e como a probabilidade dos seus membros continuarem a trabalhar juntos no futuro (Hackman, 1987; Rousseau \& Aubé, 2010); a qualidade da experiência grupal, que diz respeito à qualidade do clima social dentro da equipe (Aubé \& Rousseau, 2005; McGrath, 1991). Dada a pertinência e relevância da temática da liderança no contexto das organizações, este estudo adquire uma importância significativa, uma vez que permite não só contribuir para clarificar os mecanismos através dos quais a liderança se traduz em ganhos grupais, mas também para possibilitar a gestores e líderes de equipes o desenvolvimento de estratégias que possam dar vantagem competitiva às organizações.

\section{Liderança transformacional}

A liderança transformacional pode ser definida como um estilo adaptável e flexível de liderança em que o líder incentiva os seus colaboradores a fazerem mais do que originalmente 
esperavam fazer, alargando e mudando os seus interesses e gerando consciência e aceitação dos propósitos e missão do grupo (Bass, 1985; Bass, Avolio, Jung, \& Berson, 2003). O foco dos líderes transformacionais situa-se, assim, nas motivações coletivas, procurando mobilizar os interesses e as energias dos colaboradores em direção a um objetivo comum. Neste cotexto, a liderança transformacional pode ser entendida como um processo de influência capaz de mover os sistemas sociais com o objetivo de tornar os colaboradores agentes de mudança e atores principais no processo de desenvolvimento da organização (Bass, 1985). De acordo com Bass (1985; 1999), a liderança transformacional é um conceito multidimensional que se caracteriza pela presença de quatro dimensões as quais se encontram interligadas entre si: (a) influência idealizada, (b) motivação inspiradora, (c) estimulação intelectual e (d) consideração individual (Bass \& Avolio, 1994; Dust, Resick, \& Mawritz, 2014).

Recentemente, tendo por base abordagens anteriores (Bass \&Avolio, 1994; Podsakoff, MacKenzie, Moorman, \& Fetter, 1990), Carless, Wearing e Mann (2000) apresentam sete comportamentos característicos de um líder transformacional: (1) comunica uma visão (desen-volve uma imagem do futuro da organização e comunica-a); (2) desenvolve os membros da equipe (diagnostica as necessidades e capacidades de cada colaborador e expressa um interesse individual em cada um); (3) fornece suporte (apoia os colaboradores no alcance dos objetivos através de um trabalho de equipe coordenado); (4) dá empowerment (dá autoridade aos colaboradores para implementarem políticas e apoia-os nas suas decisões); (5) é inovador (utiliza estratégias não convencionais no alcance dos objetivos); (6) lidera pelo exemplo (adota comportamentos que são congruentes com as atitudes e valores que defende); e (7) tem carisma (inspira os colaboradores a transcender os interesses individuais, desenvolvendo uma consciência coletiva e guiando o grupo em direção ao alcance de objetivos extraordinários) (Bass, 1985; Conger \& Kanungo, 1988; Podsakoff et al., 1990; Yukl, 2010).
A investigação na área da liderança transformacional em contexto grupal aponta no sentido de que aquele estilo de liderança está positivamente associado com a eficácia das equipes, encontrando-se essa relação bem estabelecida na literatura (Judge \& Piccolo, 2004; Rico, de la Hera, \& Tabernero, 2011; Yukl, 2010). Com efeito, múltiplos estudos documentam o impacto positivo da liderança transformacional sobre diferentes resultados (ou critérios de eficácia), dos quais são exemplo a satisfação (Bass, 1999; Braun, Peus, Weisweiler, \& Frey, 2013; Judge \& Piccolo, 2004), o desempenho (Schaubroeck, Lam, \& Peng, 2011; Wang \& Howell, 2010; Wang, Oh, Courtright, \& Colbert, 2011) ou a inovação (Keller, 1992; Li, Mitchell \& Boyle, 2015; Mumford, Ginamarie, Scott, Gaddis, \& Strange, 2002).

Além dos efeitos diretos que a liderança transformacional produz sobre a eficácia grupal, a literatura também sugere que essa relação ocorre, igualmente, por via de variáveis (processos e/ou estados emergentes de grupo) que desempenham um papel mediador (Choi, Goh, Adam, \& Tan, 2016). Os comportamentos de suporte expressos pelos membros do grupo uns aos outros, quer porque são influenciados pela ação do líder quer pelos efeitos que produzem sobre a eficácia grupal, constituem, entre as referidas variáveis, um dos processos grupais que poderá mediar a relação entre a liderança transformacional e a eficácia.

\section{Liderança transformacional e comportamentos de suporte}

Aubé e Rousseau (2005, p.193) definem comportamentos de suporte "como o grau em que os membros da equipa voluntariamente prestam apoio uns aos outros, quando necessário, durante a realização da tarefa". O conceito de comportamentos de suporte procura refletir o suporte instrumental e emocional existente entre os membros da equipe. O suporte emocional remete o conjunto de ações que esses membros realizam, a fim de valorizar e auxiliar outrem ao reforçar o seu sentimento de autoestima (proporcionar incentivo 
e feedback), ao passo que o suporte instrumental envolve os diferentes tipos de ajuda tangível que essas pessoas fornecem umas às outras (ajudar nas tarefas complexas) (Aubé \& Rousseau, 2005; Tardy, 1985).

O interesse generalizado pelo estudo dos comportamentos de suporte deriva das consequências que o referido processo comportamental acarreta para as equipes e/ou organizações. A sua importância no contexto organizacional torna premente o estudo das condições que conduzem à sua emergência. Dada a centralidade do líder no grupo e a autoridade formal que possui, a forma como se comporta e atua vai influenciar o modo como a equipe funciona, nomeadamente, no que diz respeito aos comportamentos que os membros manifestam (Dimas, Rebelo, \& Lourenço, 2016; Volmer, 2012). Essa diferença de estatuto torna os comportamentos dos líderes particularmente salientes para os membros do grupo (Edmondson, 1999).

Um dos comportamentos que caracterizam o líder transformacional é o fato de fornecer suporte à equipe, aconselhando, apoiando e prestando atenção às necessidades grupais (Carless et al., 2000). Com efeito, os líderes transformacionais estabelecem objetivos desafiantes e encorajam os membros do grupo a aceitá-los e colocá-los em prática, dando-Ihes feedback positivo e reconhecendo os sucessos alcançados, mas também ajudando a desbloquear as dificuldades encontradas (Bass et al., 2003). Desta forma, os comportamentos de suporte adotados pelo líder transformacional podem funcionar como um exemplo, um modelo de referência para os membros do grupo, encorajando-os a adotarem, também, comportamentos de apoio para com os colegas (Podsakoff \& MacKenzie, 1997). Com efeito, à luz da teoria social cognitiva, os indivíduos aprendem a observar os outros e tendem a reproduzir os comportamentos que conduzem a consequências positivas (Bandura, 1986). Assim, ao exibir comportamentos de apoio, o líder transformacional age como modelo, é observado fornecendo suporte e, através do processo de aprendizagem vicariante (Bandura, 1986; Rousseau, Aubé, \& Tremblay, 2013), os mem- bros aprendem esses comportamentos e tendem a reproduzi-los em suas interações.

Além disso, ao promover o comprometimento dos membros com os objetivos grupais estabelecidos (Lim \& Ployhart, 2004), o líder transformacional propicia não só a contribuição de todos os componentes da equipe para a concretização das tarefas, como também estimula o apoio mútuo, de forma a alcançar o propósito comum mobilizador. Considerando o que precede, formulou-se a se-guinte hipótese de investigação: Hipótese 1: a liderança transformacional está relacionada positivamente com os comportamentos de suporte das equipes de trabalho.

\section{Comportamentos de suporte e eficácia grupal}

No contexto organizacional, as equipes executam uma multiplicidade de tarefas que são imprescindíveis para o sucesso (Koning \& Van Kleef, 2015). Quando os membros da equipe adotam comportamentos de suporte (ajudam os novos colegas de trabalho a tornarem-se mais produtivos) aumentam a eficácia do grupo de trabalho ou da unidade (MacKenzie, Podsakoff, \& Fetter, 1993), na medida em que possibilitam a transferência de conhecimento no seio organizacional (Koning \& Van Kleef, 2015). Os membros da equipe podem completar as respectivas tarefas, que dificilmente realizariam de forma individual, através do apoio mútuo, promovendo não só a integração das suas contribuições (Erez, Lepine, \& Elms, 2002), como também a melhoria do desempenho da equipe (Campion, Medsker, \& Higgs, 1993).

Para além dos efeitos ao nível do desempenho, evidências empíricas revelam que o suporte recíproco entre os membros pode proporcionar a melhoria do clima social intragrupo - qualidade da experiência grupal - e, por conseguinte, pode aumentar a autoestima, reforçar o moral ou propiciar uma sensação de afiliação (Podsakoff \& MacKenzie, 1997; I.G. Sarason, B.R. Sarason, \& Shearin, 1986). Além disso, esse apoio mútuo entre os membros para lidar de forma assertiva 
com as mudanças internas e externas ao contexto organizacional (Heaney, Price, \& Rafferty, 1995) pode contribuir para o aumento da viabilidade da equipe. Desta forma, de acordo com diversos estudos empíricos (Campion et al., 1993; Walz \& Niehoff, 1996), os comportamentos de suporte exercem um forte e consistente impacto sobre a eficácia grupal (Podsakoff \& MacKenzie, 1997). Apresenta-se, assim, a seguinte hipótese de inves-tigação: Hipótese 2: os comportamentos de suporte das equipes de trabalho estão relacionados positivamente com o desempenho da equipe (a); sua viabilidade (b); a qualidade da experiência grupal (c), e os processos de melhoria da equipe (d).

\section{Liderança transformacional, comportamentos de suporte e eficácia grupal}

Em conformidade com a Hipótese 1, o comportamento do líder transformacional pode ter um impacto positivo sobre as ações dos membros da equipe. Esse impacto poderá repercutir na transmissão dos comportamentos de suporte dos líderes para os membros da equipe através do processo de modelação. É esperado que os líderes que utilizem repetidamente comportamentos de suporte influenciem a conduta dos membros da equipe, induzindo-os a adotar esses comportamentos. Por outro lado, de acordo com as evidências empíricas supracitadas, os comportamentos de suporte estão fortemente relacionados com a eficácia da equipe. Desta forma, argumenta-se que o efeito da liderança transformacional sobre os resultados grupais poderá ocorrer de forma indireta, através do seu efeito ao nível dos comportamentos de suporte. Neste sentido, o presente estudo pretende testar o papel mediador dos mesmos sobre a relação entre a liderança transformacional e a eficácia da equipe. Formula-se, assim, a seguinte hipótese de investigação: Hipótese 3: os comportamentos de suporte das equipes de trabalho medeiam a relação entre a liderança transformacional e o desempenho da equipe (a); a viabilidade da equipe (b); a qualidade da experiência grupal (c), e os processos de melhoria da equipe (d).

\section{Método}

\section{Procedimentos}

A amostra do presente estudo é constituída por grupos/equipes de trabalho de áreas de atividade distintas, assim como de diferentes organizações e setores de atividade. Todas as equipes são compostas por três ou mais elementos, que se reconhecem e são reconhecidos como equipe e que interagem regularmente, de forma interdependente, tendo em vista a prossecução de um alvo comum mobilizador (Dimas et al., 2016).

Num primeiro momento, o contato inicial com cada organização efetuou-se através de comunicação oral ou escrita. Após obtido o consentimento para realização da investigação foi agendada a data para aplicação dos questionários. Em cada organização era necessário recolher dois tipos de informação: os questionários dos membros dos grupos e os questionários dos líderes. Os membros foram inquiridos a respeito da liderança transformacional, dos comportamentos de suporte e da qualidade da experiência grupal; aos líderes foi pedido que avaliassem o desempenho da equipe, sua viabilidade e os processos de melhoria.

A amostra é composta por 117 grupos de trabalho de diferentes setores de atividade (indústria, proteção civil, consultoria), com 9 elementos, em média, por grupo (Média $[M d]=5$, Desvio-Padrão $[D P]=9,15)$. As organizações participantes são, maioritariamente, médias (33,6\%) e grandes (30,3\%). As atividades mais representadas na amostra correspondem à prestação de serviços $(21,4 \%)$, seguida do comércio $(18,8 \%)$ e da produção (15,2\%).

Os membros das equipes ( $N=653)$ são, em sua maioria, do sexo feminino $(51,6 \%)$ e têm idades compreendidas entre 18 e 67 anos $(M=35,49$, $D P=9,71)$. No que diz respeito às habilitações 
acadêmicas, 38,8\% tem o ensino secundário e $26,5 \%$ tem estudos superiores. O tempo de permanência médio dos membros das equipes na organização é de, aproximadamente, 9 anos $(D P=9,10)$, e o de permanência na própria equipe é de 5 anos $(D P=5,58)$. Por fim, a interação face-a-face diária de cada colaborador com os elementos da sua equipe é de, aproximadamente, 5 horas $(D P=2,75)$.

Os líderes da equipe ( $N=122)$ são maioritariamente do sexo masculino $(67,0 \%)$ e têm idades compreendidas entre 18 e 65 anos $(M=39,29, D P=9,05)$, sendo que $47,4 \%$ tem o ensino secundário e 34,2\% tem estudos superiores. O tempo de permanência médio dos líderes na organização é de, aproximadamente, 13 anos $(D P=7,76)$, e na equipe é de 7 anos $(D P=5,95)$.

Todas as medidas usadas na presente investigação foram adaptadas para a língua portuguesa em estudos anteriores, nos quais foram identificadas evidências quanto aos seus níveis de validade. As escalas de resposta são de tipo Likert de 5 pontos, em que 1 significa "quase não se aplica" e 5 "aplica-se quase totalmente" (com exceção da qualidade da experiência grupal em que 1 significa "discordo fortemente" e 5 "concordo fortemente" e do desempenho grupal em que 1 significa "muito baixo" e 5 "muito alto").

Liderança transformacional: para avaliar a liderança transformacional utilizou-se a escala Global Transformational Leadership (GTL) de Carless et al. (2000), adaptada para a língua portuguesa por van Beveren (2015). A escala GTL é composta por sete itens, correspondendo cada um aos sete comportamentos de liderança transformacional presentes no modelo de Carless et al. O estudo da consistência interna da escala apresentou resultados bastantes satisfatórios, na medida em que o alpha de Cronbach encontrado foi de 0,96.

Comportamentos de Suporte: os comportamentos de suporte foram analisados com base na escala, composta por cinco itens, desenvolvida por Aubé e Rousseau (2005), adaptada para a língua portuguesa por Pessoa (2016), sendo que o alpha de Cronbach encontrado no presente estudo foi de 0,93 .

Eficácia Grupal: conforme já referido, a eficácia grupal foi avaliada através de quatro critérios: desempenho da equipe, sua viabilidade, seus processos de melhoria e qualidade da experiência grupal. Os três primeiros critérios mencionados são avaliados pelo líder e o último pelos colaboradores. Uma medida de desempenho da equipe desenvolvida por Rousseau e Aubé (2010), composta por cinco itens, foi adaptada para a língua portuguesa por Albuquerque (2016), tendo-se obtido, na presente amostra, um alpha de Cronbach de 0,83.

Para avaliar a viabilidade da equipe foi usada uma escala de quatro itens desenvolvida por Aubé e Rousseau (2005), adaptada para a língua portuguesa por Albuquerque (2016), sendo que o alpha de Cronbach obtido para a escala no presente estudo foi de 0,72.

Os processos de melhoria da equipe foram avaliados segundo uma escala de cinco itens desenvolvida por Rousseau e Aubé (2010), adaptada para a língua portuguesa por Albuquerque (2016), apresentando a escala, na presente amostra, um alpha de Cronbach de 0,86.

A escala que avalia a qualidade da experiência grupal contém três itens desenvolvidos por Aubé e Rousseau (2005), adaptados para a língua portuguesa por Albuquerque (2016), sendo que o alpha de Cronbach encontrado no presente estudo foi de 0,94.

Variável de controle: a dimensão da equipe foi incluída como variável de controle, uma vez que diversas investigações têm demonstrado que o efeito dos processos e das condições da equipe sobre a eficácia grupal é influenciado pelo número de colaboradores da mesma (Barrick, Stewart, Neubert, \& Mount, 1998; Mohammed \& Angell, 2004). Para obter a dimensão da equipe, os líderes foram questionados quanto ao seu número de membros. A opção por esse procedimento, em detrimento da consulta a registos formais da organização, permitiu agilizar processos de coleta de informação, bem como garantir informação de "terreno" atualizada. 


\section{Considerações éticas}

No processo de coleta de dados, as demais normas éticas que legitimam a investigação na área da Psicologia foram cumpridas (a) ao garantir a confidencialidade e o anonimato de todos os dados recolhidos; (b) ao assegurar as condições que permitem e garantem o consentimento informado das pessoas participantes, dado o caráter voluntário da investigação; (c) ao recusar a entrega de dados e resultados individuais referentes a trabalhadores da organização participante ou a outras organizações da amostra; (d) ao efetuar a coleta de dados de modo a causar o mínimo transtorno possível à organização e aos seus colaboradores e (e) ao não disponibilizar, em circunstância alguma, a lista de endereços de e-mail fornecida para aplicação do questionário online.

\section{Resultados}

\section{Procedimentos de análise de dados}

Para o teste das hipóteses, foram consideradas apenas 100 equipes de trabalho, pelo fato de terem sido eliminadas aquelas em que o número de membros que respondeu ao questionário foi inferior a $60 \%$. Da amostra dos líderes, foi ainda necessário eliminar as respostas que correspondiam a equipes que tinham sido retiradas da amostra. Desta forma, a amostra dos líderes passou a contar com 95 pessoas (houve cinco casos em que pelo menos $60 \%$ dos membros respondeu ao questionário, mas o líder não o fez; optou-se, contudo, por mantê-las na amostra, visto que uma das variáveis critério, a qualidade da experiência grupal, foi avaliada pelos membros).

Apesar de os dados relativos à liderança transformacional, aos comportamentos de suporte e à qualidade da experiência grupal terem sido recolhidos ao nível individual, o estudo se centra no nível de análise grupal. Dessa maneira, houve a necessidade de proceder à agregação das respostas dos membros das equipes através do cálculo das pontuações médias obtidas nas respectivas escalas.
Para justificar a agregação dos dados, foi calculado o Índice de Desvio Médio (Índice $A D_{M}$ ), desenvolvido por Burke, Finkelstein e Dusig (1999). Seguindo as indicações dos autores, utilizou-se o critério de $A D_{M} \leq 0,83$, para agregar, com confiança, as respostas individuais ao nível da equipe. Os valores médios de $A D_{M}$ obtidos foram os seguintes: $0,56(D P=0,26)$ para a liderança transformacional, $0,57(D P=0,25)$ para os comportamentos de suporte e $0,47(D P=0,27)$ para a qualidade da experiência grupal. Adicionalmente, para justificar a agregação dos dados, foram calculados o Intraclass Correlation Coefficient (ICC) 1 e o ICC 2 (Bliese, 2000). Os valores do ICC 1 encontrados para a liderança transformacional, comportamentos de suporte e qualidade da experiência grupal foram, respectivamente, $0,30,0,20$ e 0,18 . No que diz respeito ao ICC 2 , os valores encontrados para as mesmas variáveis foram, respectivamente, 0,69, 0,55 e 0,53 . No seu conjunto, os valores encontrados fundamentam a agregação dos dados ao nível grupal (Bliese, 2000; Klein \& Kozlowski, 2000).

Para testar as hipóteses de investigação, recorreu-se à regressão múltipla. A Hipótese 3 , que envolve mediação, foi testada através do método do produto dos coeficientes de MacKinnon, Lockwood, Hoffman, West e Sheets (2002), pelo seu poder estatístico e taxas reduzidas do erro tipo I. O método referido baseia-se na condução de duas equações de regressão. Na primeira, estima-se o valor de $\alpha$ que traduz a relação entre a variável independente $(X)$ e o mediador (M). Na segunda, estima-se o valor de $\beta$ que expressa a relação entre o mediador (M) e a variável critério $(Y)$, quando controlado o efeito de $X$ em $Y$, o qual é representado por $\tau$. O efeito indireto ou mediado é calculado através do produto de $\alpha \beta$. A significância do produto $\alpha \beta$ envolve ainda o cálculo de $P=Z \alpha \times Z \beta$. Considera-se que a relação entre $X$ e $Y$ é mediada por $M$ quando (a) a relação de $X$ e $M$ é estatisticamente significativa $(\alpha)$, (b) a relação de $M$ e $Y$, quando controlado o efeito de $X$, é estatisticamente significativa ( $\beta$ ) e (c) o efeito mediado $(\alpha \beta)$ é estatisticamente significativo (MacKinnon, 2008). Caso $\tau$ seja estatisticamente significativo, a mediação é parcial, no caso de não o 
ser, a mediação é total. O teste aos pressupostos da regressão (ausência de outliers uni e multivariados; ausência de multicolinearidade; normalidade, linearidade e homoscedacidade dos resíduos) revelou resultados satisfatórios não tendo sugerido a exclusão de nenhum caso ou variável (Tabachnick \& Fidell, 2001).

\section{Teste de Hipóteses}

Na Tabela 1 são apresentadas as médias, os desvios-padrão e as correlações entre todas as variáveis em estudo. Como é possível verificar, as correlações da variável de controle (dimensão da equipe) com as outras variáveis não são significativas, com exceção dos comportamentos de suporte e da qualidade da experiência grupal. Assim, seguindo as recomendações de Becker (2005) para omitir as potenciais variáveis de controle que não se correlacionam com as variáveis critério, a dimensão da equipe foi apenas considerada nas análises relativas aos comportamentos de suporte $(\mathrm{H} 1)$ e à qualidade da experiência grupal $(\mathrm{H} 2 \mathrm{C}$ e $\mathrm{H} 3 \mathrm{c})$.

A Hipótese 1, que prevê uma relação positiva entre a liderança transformacional e os comportamentos de suporte, foi suportada ( $\alpha=0,65$, $p<0,001$ ) (Tabela 2). Os resultados das regressões múltiplas que permitiram testar as hipóteses restantes ( $\mathrm{H} 2$ e H3) são apresentados nas Tabelas 3 e 4. Como é possível observar na Tabela 3, na regressão múltipla com a liderança transformacional e os comportamentos de suporte como variáveis preditoras e o desempenho como variável critério, há um efeito positivo significativo dos comportamentos de suporte no desempenho da equipe $(\beta=0,43, p$ $=0,001)$, corroborando, assim, H2a. A hipótese H3a, que considera os comportamentos de suporte como

Tabela 1

Análise da correlação das variáveis em estudo

\begin{tabular}{|c|c|c|c|c|c|c|c|c|c|c|}
\hline Variáveis & $\mathrm{N}$ & $M$ & $D P$ & 1 & 2 & 3 & 4 & 5 & 6 & 7 \\
\hline 1. Liderança transformacional & 100 & 3,92 & 0,63 & 1 & & & & & & \\
\hline 2. Comportamentos de suporte & 100 & 3,93 & 0,56 & $0,70^{* * *}$ & 1 & & & & & \\
\hline 3. Qualidade da experiência grupal & 100 & 4,04 & 0,53 & $0,61^{* * *}$ & $0,82^{* * *}$ & 1 & & & & \\
\hline 4. Desempenho da equipe & 95 & 4,03 & 0,59 & $0,34^{* *}$ & $0,46^{* * *}$ & $0,35^{* *}$ & 1 & & & \\
\hline 5. Viabilidade da equipe & 95 & 4,06 & 0,55 & $0,41^{* * *}$ & $0,43^{* * *}$ & $0,36^{* * *}$ & $0,62^{* * *}$ & 1 & & \\
\hline 6. Processos de melhoria da equipe & 95 & 3,86 & 0,63 & $0,36^{* * *}$ & $0,46^{* * *}$ & $0,39^{* * *}$ & $0,68^{* * *}$ & $0,59^{* * *}$ & 1 & \\
\hline 7. Dimensão da equipe & 87 & 7,93 & 9,15 & $-0,15$ & $-0,25^{*}$ & $-0,32^{* *}$ & $-0,10$ & 0,01 & $-0,17$ & 1 \\
\hline
\end{tabular}

Nota: ${ }^{*} p<0,05 ;{ }^{* *} p<0,01 ;{ }^{* * *} p<0,001$.

$M$ : Média; $D P$ : Desvio-Padrão.

Tabela 2

Resultados da Análise da Regressão Hierárquica da Liderança transformacional como preditora dos Comportamentos de Suporte

\begin{tabular}{|c|c|c|c|c|c|}
\hline Variáveis & $B$ & $E P B$ & $\beta$ & $R^{2}$ & $\Delta R^{2}$ \\
\hline Passo 1 & & & & $0,06^{*}$ & \\
\hline Número de elementos da equipe & $-0,01$ & 0,01 & $-0,25^{*}$ & & \\
\hline Passo 2 & & & & $0,48^{* * *}$ & $0,42^{* * *}$ \\
\hline Número de elementos da equipe & $-0,01$ & 0,00 & $-0,15$ & & \\
\hline Liderança transformacional & 0,54 & 0,07 & $0,65^{* * *}$ & & \\
\hline
\end{tabular}

Nota: ${ }^{*} p<0,05 ;{ }^{* *} p<0,01 ;{ }^{* * *} p<0,001$.

22 EPB: Erro Padrão do coeficiente de regressão. 
variável mediadora na relação entre a liderança transformacional e o desempenho da equipe, também foi corroborada, uma vez que (a) a liderança transformacional apresenta uma relação positiva significativa com os comportamentos de suporte $(\alpha=0,65, p<0,001)$, (b) os comportamentos de suporte apresentam uma relação positiva significativa com o desempenho da equipe $(\beta=0,43, p=0,001)$, após o controle da liderança transformacional, (c) e o efeito mediado estimado $(\alpha \beta=0,28)$ foi estatisticamente significativo $(P=Z \alpha \times Z \beta=30,75, p<0,01)$. Como é possível verificar, os comportamentos de suporte medeiam totalmente a relação entre a liderança transformacional e o desempenho da equipe, dado que o efeito direto da liderança transformacional no desempenho da equipe não é significativo $(\tau=0,05, p=0,705)$.
Na regressão múltipla com a liderança transformacional e os comportamentos de suporte como variáveis preditoras e a viabilidade da equipe como variável critério, como é possível observar na Tabela 3, há um efeito positivo significativo dos comportamentos de suporte na viabilidade da equipe $(\beta=0,29, p=0,026)$, corroborando, assim, a H2b. A H3b, que considera os comportamentos de suporte como variável mediadora na relação entre a liderança transformacional e a viabilidade da equi-pe, também foi corroborada, uma vez que (a) a liderança transformacional apresenta uma relação positiva significativa com os comportamentos de suporte $(\alpha=0,65, p<0,001)$, (b) os comportamentos de suporte apresentam uma relação positiva significativa com a viabilidade da equipe

Tabela 3

Resultados da Análise de Regressão Múltipla com os Comportamentos de Suporte como Preditor do Desempenho Grupal, da Viabilidade do Grupo e dos Processos de Melhoria do Grupo

\begin{tabular}{|c|c|c|c|c|}
\hline Modelo & B & $E P B$ & $\beta$ & $R^{2}$ \\
\hline Variável dependente: Desempenho Grupal & & & & $0,21^{* * *}$ \\
\hline Liderança Transformacional & 0,05 & 0,12 & 0,05 & \\
\hline Comportamentos de Suporte & 0,44 & 0,13 & $0,43^{* *}$ & \\
\hline Variável dependente: Viabilidade do Grupo & & & & $0,21^{* * *}$ \\
\hline Liderança Transformacional & 0,18 & 0,11 & 0,20 & \\
\hline Comportamentos de Suporte & 0,28 & 0,12 & $0,29^{*}$ & \\
\hline Variável dependente: Processos de Melhoria do Grupo & & & & $0,22^{* * *}$ \\
\hline Liderança Transformacional & 0,08 & 0,13 & 0,08 & \\
\hline Comportamentos de Suporte & 0,45 & 0,14 & $0,41^{* *}$ & \\
\hline
\end{tabular}

Nota: ${ }^{*} p<0,05 ;{ }^{* *} p<0,01 ;{ }^{* * *} p<0,001$.

EPB: Erro Padrão do coeficiente de regressão.

Tabela 4

Resultados da Análise da Regressão Hierárquica dos Comportamentos de Suporte como preditor da Qualidade da Experiência Grupal

\begin{tabular}{|c|c|c|c|c|c|}
\hline Variáveis & B & $E P B$ & $\beta$ & $R^{2}$ & $\Delta R^{2}$ \\
\hline Passo 1 & & & & $0,10^{* *}$ & \\
\hline Número de elementos da equipe & $-0,02$ & 0,01 & $-0,32^{* *}$ & & \\
\hline Passo 2 & & & & $0,69^{* * *}$ & $0,58^{* * *}$ \\
\hline Número de elementos da equipe & $-0,01$ & 0,00 & $-0,12$ & & \\
\hline Liderança transformacional & 0,05 & 0,07 & 0,06 & & \\
\hline Comportamentos de Suporte & 0,77 & 0,09 & $0,75^{* * *}$ & & \\
\hline
\end{tabular}

Nota: ${ }^{*} p<0,05 ;{ }^{* *} p<0,01 ;{ }^{* * *} p<0,001$. 
$(\beta=0,29, p=0,026)$, após o controle da liderança transformacional, (c) e o efeito mediado estimado $(\alpha \beta=0,19)$ foi estatisticamente significativo $(P=Z \alpha$ $x Z \beta=22,5, p<0,01)$. Como é possível verificar, os com-portamentos de suporte medeiam totalmente a relação entre a liderança transformacional e a viabilidade da equipe, dado que o efeito direto da liderança transformacional na viabilidade da equipe não é significativo $(\tau=0,20, p=0,122)$.

$\mathrm{Na}$ regressão múltipla com a liderança transformacional e os comportamentos de suporte como variáveis preditoras e a qualidade da experiência grupal como variável critério, como é possível observar na Tabela 4, há um efeito positivo significativo dos comportamentos de suporte na qualidade da experiência grupal ( $\beta=0,75, p<0,001)$, corroborando, assim, a $\mathrm{H} 2 \mathrm{c}$. A H3C, que considera os comportamentos de suporte como variável mediadora na relação entre a liderança transformacional e a qualidade da experiência grupal, também foi corroborada, uma vez que (a) a liderança transformacional apresenta uma relação positiva significativa com os comportamentos de suporte $(\alpha=0,65, p<0,001)$, (b) os comportamentos de suporte apresentam uma relação positiva significativa com a qualidade da experiência grupal $(\beta=0,75, p<0,001)$, após o controle da liderança transformacional, (c) e o efeito mediado estimado $(\alpha \beta=0,49)$ foi estatisticamente significativo $(P=Z \alpha \times Z \beta=77,39, p<0,01)$. Dado que o efeito direto da liderança transformacional na qualidade da experiência grupal não é significativo $(\tau=0,06, p=0,456)$, pode-se dizer que existe uma mediação total.

Na regressão múltipla com a liderança transformacional e os comportamentos de suporte como variáveis preditoras e os processos de melhoria da equipe como variável critério, como é possível observar na Tabela 3, há um efeito positivo significativo dos comportamentos de suporte nos processos de melhoria da equipe ( $\beta=0,41, p=0,002)$, corroborando, assim, a $\mathrm{H} 2 \mathrm{~d}$. A H3d, que considera os comportamentos de suporte como variável mediadora na relação entre a liderança transformacional e os processos de melhoria da equipe, também foi corroborada, uma vez que (a) a liderança transformacional apresenta uma relação positiva significativa com os comportamentos de suporte $(\alpha=0,65, p<0,001)$, (b) os comportamentos de suporte apresentam uma relação positiva significativa com os processos de melhoria da equipe $(\beta=0,41, p=0,002)$, após o controle da liderança transformacional, (c) e o efeito mediado estimado $(\alpha \beta=0,27)$ foi estatisticamente significativo $(P=Z \alpha \times Z \beta=27,22, p<0,01)$. Como é possível verificar, os comportamentos de suporte medeiam totalmente a relação entre a liderança transformacional e os processos de melhoria da equipe, dado que o efeito direto da liderança transformacional nos processos de melhoria da equipe não é significativo $(\tau=0,08, p=0,543)$.

\section{Discussão}

No presente estudo, foi objetivo central investigar o papel mediador dos comportamentos de suporte na relação entre a liderança transformacional e a eficácia grupal (medida pelos critérios desempenho, viabilidade, qualidade da experiência grupal e os processos de melhoria). Os resultados da Hipótese 1 baseada na literatura de aprendizagem vicariante (Bandura, 1986), a qual previu que os membros da equipe, ao observar e experimentar comportamentos de suporte dos líderes, poderiam também adotar comportamentos de suporte, apoiam este efeito positivo esperado. Perante um líder transformacional que utiliza escuta ativa, age como mentoring e fornece feedback regular, isto é, utiliza comportamentos de suporte, a adoção desses mesmos comportamentos por parte dos membros da equipe torna-se mais provável, uma vez que, o líder enquanto modelo determina a aprendizagem dos mesmos (Bass, 1999).

A Hipótese 2, relativa à relação entre os comportamentos de suporte e a eficácia da equipe, também foi corroborada. Os resultados indicam que os comportamentos de suporte influenciam positivamente as várias dimensões da eficácia da equipe consideradas. Estes resultados sugerem que o apoio voluntário entre os membros da equipe durante o desenvolvimento das tarefas potencializa os resultados de desempenho de 
tarefa e a capacidade de os membros introduzirem melhorias, tendo em vista o desenvolvimento de novas práticas de trabalho. De igual modo, o apoio voluntário disponibilizado pelos membros aumenta a capacidade destes para lidar com os problemas que podem afetar a sua estabilidade social (Barrick et al., 1998) e permite reforçar a qualidade do clima social intraequipe (Aubé \& Rousseau, 2005).

Este estudo representa a primeira tentativa de examinar o efeito mediador dos comportamentos de suporte na relação entre a liderança transformacional e os critérios de eficácia da equipe. Como previsto na Hipótese 3 , os resultados indicam que os comportamentos de suporte medeiam totalmente a relação entre a liderança transformacional e o desempenho da equipe, sua a viabilidade, a qualidade da experiência grupal e os processos de melhoria. Estes resultados sugerem, assim, que a adoção de um estilo transformacional por parte de líderes de equipes constitui uma mais valia para o desenvolvimento de comportamentos de suporte no seio da equipe, os quais permitem potencializar a eficácia grupal em diferentes dimensões.

\section{Considerações Finais}

O presente estudo evidencia a importância dos comportamentos de suporte enquanto mediadores da relação entre a liderança transformacional e a eficácia da equipe. Em termos específicos, os resultados sugerem que, visando melhorar a eficácia da equipe, é vantajoso que a ação dos líderes e dos demais executivos assuma caraterísticas transformacionais, pois este estilo contribui para a promoção dos comportamentos de suporte.

Assim, esta pesquisa contribui para enriquecer o conhecimento acerca do funcionamento grupal e, em particular, acerca dos efeitos potenciais da liderança transformacional e dos comportamentos de suporte na eficácia da equipe. Apesar de possuir a mais valia de ter incidido em equipes reais de diferentes organizações e setores de atividade, apresenta algumas limitações que precisam ser assinaladas. Em primeiro lugar, todas as variáveis em análise foram medidas através do método do questionário. Embora esse método apresente múltiplas vantagens (baixo custo, facilidade de análise e familiarização com o método), uma abordagem multimétodo pode produzir dados mais ricos. Em segundo lugar, embora os dados tenham sido recolhidos por meio de duas fontes de avaliação (membros da equipe e superiores imediatos), o que contribui para um diagnóstico mais abrangente da eficácia e permite a diminuição dos efeitos da variância do método comum (Conway \& Lance, 2010), a medida da liderança transformacional foi de natureza perceptiva. Embora essa limitação tenha sido atenuada com a agregação dos dados ao nível grupal, avaliou-se a percepção que os membros de uma equipe têm relativamente aos comportamentos do seu líder e não os comportamentos em si.

Além disso, o desempenho, a viabilidade e os processos de melhoria foram avaliados pelos superiores imediatos, constituindo indicadores subjetivos dos resultados da equipe. Para apresentar uma imagem favorável da mesma, os superiores hierárquicos podem ter fornecido classificações inflacionadas, sendo influenciados pelo viés da desejabilidade social (Rousseau \& Aubé, 2010). Importa notar, no entanto, que no contexto organizacional são os supervisores que estão em melhor posição para fornecer informação sobre os critérios da eficácia de uma equipe.

Pesquisas futuras devem, contudo, tentar superar essa limitação, integrando, por exemplo, indicadores objetivos - embora, segundo estudos anteriores, os indicadores subjetivos estejam correlacionados positivamente com os objetivos (Ancona \& Caldwell, 1992). Finalmente, o presente estudo teve por base um desenho transversal, o que impossibilita a evidência direta das relações causais entre as variáveis em estudo. Considerando os resultados positivos do estudo, é desejável que pesquisas futuras adotem design longitudinal, de forma a obter conclusões sobre a causalidade das relações (Maxwell \& Cole, 2007).

Várias implicações conceituais e de intervenção emergem do estudo realizado. Ao nível conceitual, os resultados acrescentam conhecimento 
ao mostrarem que (a) os líderes transformacionais têm um papel relevante nos comportamentos de suporte e (b) estes, por sua vez, constituem fatores importantes que potenciam os resultados de uma equipe. Em conjunto com achados de outros estudos (Dionne, Yammarino, \& Spangler, 2004; Gross, 2014; Schlechter \& Strauss, 2008; Zhu et al., 2013), os quais sugerem que aspetos como a confiança, a coesão ou o comprometimento com a equipe possam ser estimulados pela ação do líder, os resultados do presente artigo reforçam a importância de dar continuidade a estudos que, na perspetiva aqui adotada, analisem os efeitos mediadores de diferentes processos/estados emergentes de grupo na relação entre liderança e eficácia na investigação sobre o funcionamento e a eficácia grupal.

No que diz respeito à intervenção, os resultados do estudo sugerem que os líderes devem adotar comportamentos de apoio, a fim de promoverem os comportamentos de suporte dos membros da equipe. Atender as preocupações e necessidades da sua equipe, contribuir para o desenvolvimento pessoal de cada membro (Yukl, 2010) e atribuir tarefas desafiadoras, facilitará a emergência e desenvolvimento de comportamentos de apoio entre os membros, contribuindo, igualmente, para que estes se superem, com reflexos nos resultados grupais. Mostrar confiança no trabalho da equipe e fornecer feedback constituem, igualmente, boas formas de dar suporte ao grupo, através do reconhecimento público do seu trabalho (Bosch, 2013; Carless et al., 2000). Neste contexto, as organizações devem procurar implementar programas formativos que estimulem o desenvolvimento de competências transformacionais dos seus líderes de forma a potencizalizar a eficácia das suas equipes.

\section{Colaboradores}

C. I. P. PESSOA colaborou na revisão da literatura, coleta de dados, análise e interpretação dos resultados. I.D. DIMAS colaborou na concepção e desenho do estudo, análise e interpretação dos resultados, revisão e aprovação da versão final do artigo. P. R. LOURENÇO e T. REBELO colaboraram na concepção e desenho do estudo, revisão e aprovação da versão final do artigo.

\section{Referências}

Albuquerque, L. B. (2016). Team resilience and team effectiveness: Adaptation of measuring instruments (Tese de mestrado não publicada). Universidade de Coimbra,Portugal.

Ancona, D., \& Caldwell, D. (1992). Demography and design: Predictors of new product team performance. Organization Science, 3(3), 321-341.

Aubé, C., \& Rousseau, V. (2005). Team goal commitment and team effectiveness: The role of task interdependence and supportive behaviors. Group Dynamics: Theory, Research, and Practice, 9(3), 189-204.

Bandura, A. (1986). Social foundations of thought and action: A social cognitive theory. Englewood Cliffs, NJ: Prentice-Hall.

Barrick, M. R., Stewart, G. L., Neubert, M. J., \& Mount, M. K. (1998). Relating member ability and personality to work-team processes and team effectiveness. Journal of Applied Psychology, 83(3), 377-391.

Bass, B. M. (1985). Leadership and performance beyond expectations. New York: Free Press.

Bass, B. M. (1999). Two decades of research and development in transformational leadership. European Journal of Work and Organizational Psychology, 8(1), 9-32.

Bass, B. M., \& Avolio, B. J. (1994). Improving organizational effectiveness: through transformational leadership. Thousand Oaks: Sage.

Bass, B. M., Avolio, B. J., Jung, D. I., \& Berson, Y. (2003). Predicting unit performance by assessing transformational and transactional leadership. Journal of Applied Psychology, 88(2), 207-218.

Becker, T. E. (2005). Potential problems in the statistical control of variables in organizational research: A qualitative analysis with recommendations. Organizational Research Methods, 8(3), 274-289.

Bliese, P. D. (2009). Multilevel modelling in R (2.3). A brief introduction to $R$, the multilevel package and the nlme package. Los Angeles, CA: University of California. Retrieved November 5, 2015, from http://www.cran.rproject.org/doc/contrib/Bliese Multilevel.pdf

Bosch, D. (2013). The impact of transformational leadership on leader-follower work value congruence. International Journal of Business and Social Research, 3(8), 18-31.

Braun, S., Peus, C., Weisweiler, S., \& Frey, D. (2013). Transformational leadership, job satisfaction, and team performance: A multilevel mediation model of trust. The Leadership Quarterly, 24(1), 270-283.

Burke, C. S., Stagl, K. C., Klein, C., Goodwin, G. F., Salas, E., \& Halpin, S. M. (2006). What type of leadership behaviors are functional in teams? A meta-analysis. The Leadership Quarterly, 17(3), 288-307. 
Burke, M. J., Finkelstein, L. M., \& Dusig, M. S. (1999). On average deviation indices for estimating interrater agreement. Organizational Research Methods, 2(1), 49-68.

Campion, A. C., Medsker, G. J., \& Higgs, A. C. (1993). Relations between work group characteristics and effectiveness: Implications for designing effective work groups. Personnel Psychology, 46(4), 823-850.

Carless, S. A., Wearing, A. J., \& Mann, L. (2000). A short measure of transformational leadership. Journal of Business and Psychology, 14(3), 389-405.

Choi, S. L., Goh, C. F., Adam, M. B., \& Tan, O. K. (2016). Transformational leadership, empowerment, and job satisfaction: The mediating role of employee empowerment. Human Resources for Health, 14(1), 73.

Conger, J., \& Kanungo, R. (1988). The empowerment process: Integrating theory and practice. Academy of Management Review, 13(3), 471- 482.

Conway, J. M., \& Lance, C. E. (2010). What reviewers should expect from authors regarding common method bias in organizational research. Journal of Business and Psychology, 25(3), 325-334.

Day, D. V., \& Antonakis, J. (2012). Leadership: Past, present, and future. In D. V. Day \& J. Antonakis (Eds.), The nature of leadership (2nd ed., pp. 3-25). Los Angeles, CA: Sage.

Dimas, I. D., Rebelo, T., \& Lourenço, P. R. (2016). Team coaching: One more clue for fostering team effectiveness. European Review of Applied Psychology. 66(5), 233-242.

Dionne, S. D., Yammarino, F. J., \& Spangler, W. D. (2004). Transformational leadership and team performance. Journal of Organizational Change, 17(2), 177-193.

Dust, S. B., Resick, C. J., \& Mawritz, M. B. (2014). Transformational leadership, psychological empowerment, and the moderating role of mechanisticorganic contexts. Journal of Organizational Behavior, 35(3), 413-433.

Edmondson, A. (1999). Psychological safety and learning behavior in work teams. Administrative Science Quarterly, 44(2), 350-383.

Erez, A., Lepine, J. A., \& Elms, H. (2002). Effects of rotated leadership and peer evaluation on the functioning and effectiveness of self-managed teams: A quasiexperiment. Personnel Psychology, 55(4), 929-948.

Gross, R. (2014). The Impact of transformational leadership, climate and trust in cross-funtional teams. International Journal of Recent Advances in Organizational Behaviour and Decision Sciences, 1(2), 108-132.

Hackman, J. R. (1987). The design of work teams. In J. Lorsch (Ed.), Handbook of organizational behavior (pp. 315-342). Englewood Cliffs, NJ: Prentice Hall.
Hackman, J. R. (2012). From causes to conditions in group research. Journal of Organizational Behavior, 33, 428-444.

Heaney, C. A., Price, R. H., \& Rafferty, J. (1995). Increasing coping resources at work: A field experiment to increase social support, improve work team functioning, and enhance employee mental health. Journal of Organizational Behavior, 16(4), 335-352.

Ilgen, D. R., Hollenbeck, J. R., Johnson, M., \& Jundt, D. (2005). Teams in organizations: From input-processoutput models to IMOI models. Annual Review of Psychology, 56, 517-543.

Judge, T. A., \& Piccolo, R. F. (2004). Transformational and transactional leadership: A meta-analytic test of their relative validity. Journal of Applied Psychology, 89(5), 755-768.

Keller, R. T. (1992). Transformational leadership and the performance of research and development project groups. Journal of Management, 18(3), 489-501.

Klein, K. J., \& Kozlowski, S. W. (Eds.). (2000). Multilevel theory, research, and methods in organizations: Foundations, extensions, and new directions. San Francisco: Jossey-Bass.

Koning, L. F., \& Van Kleef, G. A. (2015). How leaders' emotional displays shape followers' organizational citizenship behavior. The Leadership Quarterly, 26(4), 489-501.

Kozlowski, S. W. J., \& Ilgen, D. R. (2006). Enhancing the effectiveness of work groups and teams. Psychological Science in the Public Interest, 7(3), 77-124.

Li, V., Mitchell, R., \& Boyle, B. (2015). The divergent effects of transformational leadership on individual and team innovation. Group \& Organization Management, 41(1), 66-97.

Lim, B. C., \& Ployhart, R. E. (2004). Transformational leadership: Relations to the five-factor model and team performance in typical and maximum contexts. Journal of Applied Psychology, 89(4), 610-621.

MacKenzie, S. B., Podsakoff, P. M., \& Fetter, R. (1993). The impact of organizational citizenship behavior on evaluations of salesperson performance. Journal of Marketing, 57(1), 70-80.

MacKinnon, D. P. (2008). Introduction to statistical mediation analysis. New Jersey: Mahway.

MacKinnon, D. P., Lockwood, C. M., Hoffman, J. M., West, S. G., \& Sheets, V. (2002). A comparison of methods to test mediation and other intervening variable effects. Psychological Methods, 7(1), 83-104.

Maxwell, S. E., \& Cole, D. A. (2007). Bias in cross-sectional analyses of longitudinal mediation. Psychological Methods, 12(1), 23-44. https://doi.org/10.1037/108 2-989x.12.1.23

McGrath, J. E. (1991). Time, interaction, and performance (TIP): A theory of groups. Small Group Research, 22, 147-174. https://doi.org/0803973233 
Mohammed, S., \& Angell, L. C. (2004). Surface-and deep-level diversity in workgroups; examining the moderating effects of team orientation and team process on relationship conflict. Journal of Organizational Behavior, 25(8), 1015-1039.

Mumford, M. D., Scott, G. M., Gaddis, B., \& Strange, J. M. (2002). Leading creative people: Orchestrating expertise and relationships. The Leadership Quarterly, 13(6), 705-750.

Pessoa, C. (2016). Liderança transformacional e a eficácia grupal: o papel mediador da resiliência e dos comportamentos de suporte (Tese de mestrado não-publicada). Universidade de Coimbra, Portugal.

Podsakoff, P. M., \& MacKenzie, S. B. (1997). Impact of organizational citizenship behavior on organizational performance: A review and suggestions for future research. Human Performance, 10(2), 133-151.

Podsakoff, P. M., MacKenzie, S. B., Moorman, S., \& Fetter, R. (1990). Transformational leader behaviors and their effects on followers' trust in leader, satisfaction, and organizational citizenship behaviors. The Leadership Quarterly, 1(2), 107-142.

Rico, R., de la Hera, C. M., \& Tarbenero, C. (2011). Work team effectiveness, a review of research from the last decade (1999-2009). Psychology in Spain, 15(1), 57-79.

Rousseau, V., \& Aubé, C. (2010). Team self-managing behaviors and team effectiveness: The moderating effect of task routineness. Group \& Organization Management, 35(6), 751-781.

Rousseau, V., Aubé, C., \& Tremblay, S. (2013). Team coaching and innovation in work teams: An examination of the motivational and behavioral intervening mechanisms. Leadership \& Organization Development Journal, 34(4), 344-364.

Sarason, I. G., Sarason, B. R., \& Shearin, E. N. (1986). Social support as an individual difference variable: Its stability, origins, and relational aspects. Journal of Personality and Social Psychology, 50(4), 845-855.

Schaubroeck, J., Lam, S. S. K., \& Peng, A. C. (2011). Cognition-based and affect-based trust as mediators of leader behavior influences on team performance. Journal of Applied Psychology, 96(4), 863-871.
Schlechter, A. F., \& Strauss, J. J. (2008). Leader emotional intelligence, transformational leadership, trust and team commitment: Testing a model within a team context. Journal of Industrial Psychology, 34(1), 42-53.

Tabachnick, B. G., \& Fidell, L. S. (2001). Using multivariate statistics (4th ed.). Boston: Allyn \& Bacon.

Tardy, C. H. (1985). Social support measurement. American Journal of Community Psychology, 13(2), 187-202.

Van Beveren, P. (2015). Liderança transformacional e autonomia grupal: adaptação de instrumentos de medida (Tese de mestrado não-publicada). Faculdade de Psicologia e Ciências da Educação, Coimbra, Portugal.

Volmer, J. (2012). Catching leaders' mood: Contagion effects in teams. Administrative Sciences, 2(3), 203-220.

Walz, S. M., \& Niehoff, B. P. (1996). Organizational citizenship behaviors and their effect on organizational effectiveness in limited-menu restaurants. In J. B. Keys \& L. N. Dosier (Eds.), Academy of management best papers proceedings (pp. 307-311). Briarcliff Manor, NY: Academy of Managment.

Wang, G., Oh, I. S., Courtright, S. H., \& Colbert, A. E. (2011). Transformational leadership and performance across criteria and levels: A meta-analytic review of 25 years of research. Group \& Organization Management, 36(2), 223-270.

Wang, X. H., \& Howell, J. M. (2010). Exploring the dual-level effects of transformational leadership on followers. Journal of Applied Psychology, 95(6), 1134-1144.

Yukl, G. A. (2010). Leadership in organizations (7th ed.). Upper Saddle River, NJ: Prentice Hall.

Zhu, W., Newman, A., Miao, Q., \& Hooke, A. (2013). Revisiting the mediating role of trust in transformational leadership effects: Do different types of trust make a difference? The Leadership Quarterly, 24(1), 94-105.

Recebido: agosto 24, 2016

Versão final: janeiro 27, 2017

Aprovado: fevereiro 23, 2017 